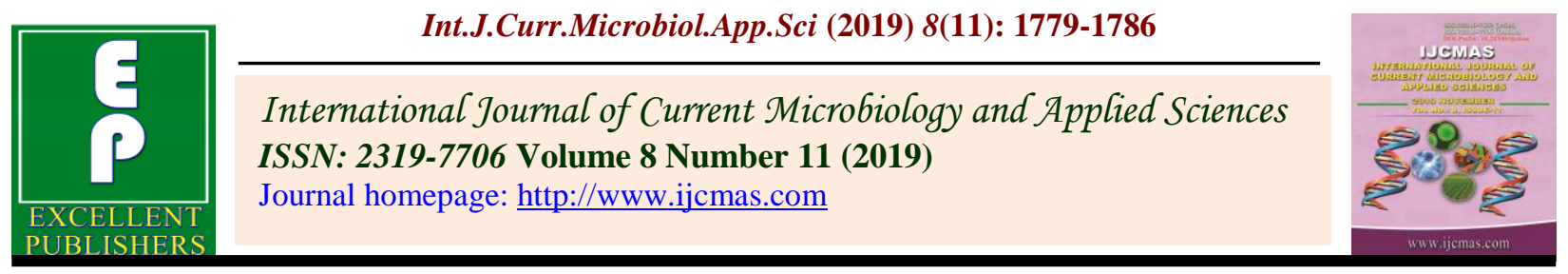

\title{
Mobile Phones of Healthcare Workers - A Boon for Communication or A Potential Reservoir of Nosocomial Pathogens?
}

\author{
Siddesh Basawaraj Sirwar ${ }^{1}$ and Javeria Firdous ${ }^{2 *}$ \\ Department of Microbiology, KBN Institute of Medical Sciences, \\ Kalaburagi, Karnataka, India \\ *Corresponding author
}

\section{A B S T R A C T}

\section{Keywords}

Mobile phones,

Nosocomial infections, Health

Care Workers

(HCWs), $70 \%$

isopropyl alcohol

Article Info

Accepted:

17 October 2019

Available Online:

10 November 2019
Hospital acquired infections are a problem in both developed and developing countries. India is ranked second in the list of number of mobile phone users in the world. Healthcare workers do use mobile phones. However they are seldom cleaned, therefore serve as potential reservoir of nosocomial pathogens: To determine the bacterial flora on mobile phones of HCWs and non HCWs; To determine the antimicrobial susceptibility pattern of microorganisms isolated from mobile phones of HCWs and non HCWs; To study the efficacy of decontamination with a single $70 \%$ isopropyl alcohol swipe. A total of 150 HCWs and 50 Non HCWs were involved in the study. The samples were collected aseptically from mobile phones before and after disinfection with $70 \%$ isopropyl alcohol using sterile cotton swab moistened with sterile normal saline. Pre- and post- disinfection swabs were inoculated onto blood agar and MacConkey agar plate, incubated aerobically at $37^{\circ} \mathrm{C}$ for 24 hours. Identification was done as per recommended standard microbiological methods. Antimicrobial susceptibility testing was performed as per CLSI guidelines. Among 150 mobile phones of HCWs, 141 yielded single or mixed growth with a total of 306 isolates. CONS (113) were prominent isolates followed by gram positive bacilli (96), MSSA (28), MRSA (24), Pseudomonas (14), Micrococcus (14), Enterococcus (10) and E. coli (7). Out of 50 Non HCWs, 15(30\%) showed growth, of which 11 were CONS \& 7 were Gram positive bacilli. Antimicrobial susceptibility testing showed multi drug resistant organisms in mobile phones of HCWs. The efficacy of decontamination was found to be $89 \%$ as only 15 mobile phones of HCWs showed growth after decontamination. We conclude that mobile phones of HCWs can act as a potential source of nosocomial infections and simple usage of alcohol disinfectant and hand washing practices can reduce the risk of infection.

\section{Introduction}

In this electronic age, mobile phones are used as a means of communication which has made our life very simple and easy going. There are almost as many mobile phones as there are humans on the planet ${ }^{1}$. India is ranked second in the list of number of mobile phone users in the world ${ }^{2}$. The greatest advantage of mobile phones compared to old telephones is that it can be used anywhere, anytime. It can be used as computer and the whole world in one's hand. It is easy to overlook its hazards to health when compared to its benefits. 
Hospital acquired infections are a problem in both developed and developing countries ${ }^{3}$. The sources of infection can be endogenous or exogenous. Exogenous sources which can serve as reservoir of infection are patients, Health care workers (HCWs), inanimate objects like stethoscopes, mobile phones, and other items present in the immediate vicinity of the patient. Over a past decade, use of mobile phones has become indispensible in the hospital. It may support certain aspects of clinical diagnosis and education. Healthcare workers do use mobile phones. Microorganisms are transferred from patients to HCWs hand while attending the patients. If HCWs use mobile phones without proper hand washing, these microorganisms can be transferred to mobile phones.

If mobiles are not cleaned and again used while attending another patient, these organisms can be transferred to other patients and can cause nosocomial infections. If the transferred organisms are antibiotic resistant strains then mobile phones can act as a potential reservoir of nosocomial pathogens. Even sharing of mobile phones between HCWs and non HCWs may directly lead to transfer of resistant strains to community. Hence the present study was done to determine the organisms associated with the mobile phones of HCWs and its susceptibility pattern, to determine efficacy of decontamination with alcohol and also to develop awareness in HCWs and non HCWs for proper handling of mobile phones.

The main objectives of this study includes to determine the bacterial flora on mobile phones of HCWs and non HCWs. Also, to determine the antimicrobial susceptibility pattern of microorganisms isolated from mobile phones of HCWs and non HCWs. And to study the efficacy of decontamination with a single $70 \%$ isopropyl alcohol swipe.

\section{Materials and Methods}

This prospective cross sectional study was conducted at Khaja Banda Nawaz teaching and general hospital dated from $1^{\text {st }}$ August to $31^{\text {st }}$ August 2019. A total of $150 \mathrm{HCWs}$ (doctors and nurses) and 50 Non HCWs (OPD patient attenders, members working in hospital canteen) were involved in the study. Participants using mobile accessories like Bluetooth devices, ear phones, etc were excluded from the study. Written informed consent was taken from the participants. The study was approved by the ethical committee of our institute.

The samples were collected aseptically from mobile phones (front, back and sides) before disinfection with alcohol using sterile cotton swab moistened with sterile normal saline. Disinfection of mobile phones was done using a single $70 \%$ isopropyl alcohol swipe. Mobile phones were allowed to dry for 10 minutes.

After 10 minutes samples were collected in the same said manner. Pre- and postdisinfection swabs were inoculated onto blood agar and MacConkey agar plate, incubated aerobically at $37^{\circ} \mathrm{C}$ for 24 hours. Identification was done as per recommended standard microbiological methods ${ }^{4}$. Methicillin resistance of isolated $S$. aureus was detected by cefoxitin disc diffusion method as per CLSI guidelines ${ }^{5}$. Antimicrobial susceptibility testing was performed on Mueller Hinton Agar using Kirby Bauer disk diffusion method as per CLSI guidelines 5 .

\section{Results and Discussion}

Among 150 mobile phones of HCWs before decontamination, 141(94.05\%) yielded single or mixed growth with a total of 306 isolates. Among 50 mobile phones of Non HCWs before decontamination, $15(30 \%)$ yielded single or mixed growth with a total of 18 
isolates (Table 1 and 2). Out of 141 positive microbial growth on mobile phones of HCWs, Coagulase negative Staphylococci (CONS) $(113 ; 80.14 \%)$ were predominant isolates followed by Gram positive bacilli (96;68.1\%), Methicillin sensitive Staphylococcus aureus (MSSA) (28;19.8\%), Methicillin resistant Staphylococcus aureus (MRSA) (24; 17\%), Pseudomonas (14; 9.9\%), Micrococcus (14; 9.9\%), Enterococcus $(10 ; 7.1 \%)$ and E. coli (7; $4.9 \%)$. Out of 15 positive microbial growth on mobile phones of Non HCWs CONS (11; $73.34 \%$ ) were predominant isolates followed by Gram positive bacilli $(7 ; 46.67 \%$ ) (Table 2).

In HCWs, 24 isolates were found in 15 mobile phones after decontamination, of which 11(73.34\%) were found to be CONS, followed by Gram positive bacilli $(7 ; 46.67 \%)$, MSSA $(4 ; 26.67 \%))$, MRSA $(2 ; 13.34 \%)$ (Table 3$)$.

In HCWs, all gram positive organisms were complete sensitive to LZ, VA and TEI except MRSA which is $79 \%$ sensitive to VA. CONS were least sensitive to AK \& E (73\%). Gram positive bacilli were least sensitive to GEN (68\%). MSSA were least sensitive to GEN \& E (50\%). MRSA were least sensitive to $\mathrm{E}$ (21\%). Micrococcus was least sensitive to CIP (64\%). Enterococcus was least sensitive to CIP (40\%) (Table 4). In HCWs, all gram negative organisms were sensitive to IMP, PI, TOB \& CL. Pseudomonas was least sensitive to AK, GEN \& CIP (36\%). E. coli were least sensitive to GEN (29\%) (Table 5). The mobile phones of Non HCWs showed growth of only gram positive organisms. CONS were $100 \%$ sensitive to AMC, CD, DO, LZ, VA\& TEI and least sensitive to CIP \& E. Gram positive bacilli is $100 \%$ sensitive to AMC, LZ, VA \& TEI and least sensitive to CIP \& E (71\%) (Table 6). The efficacy of decontamination with a single $70 \%$ isopropyl alcohol swipe was found to be $89 \%$ as only 15 mobile phones of HCWs showed growth after decontamination. There was no growth of microorganism observed in mobile phones of non HCWs after decontamination (Table 7).

The first mobile telecommunication was started in Europe in 1982 for improvement in communication system. In India, the first use of mobile phone was done in 1995. Today more people worldwide own mobile phones than have access to working toilets ${ }^{6}$.

Mobile phones are carried by the doctors and HCWs and are extensively used within the hospital premises including areas like Operation theatres and Intensive care units which may serve as reservoirs of potentially pathogenic bacteria leading to infections.

In a study it was discovered that average cell phone is dirtier than either a toilet seat or the bottom of your shoe ${ }^{7}$. Our phones are ideal breeding sites for microbes as they are kept warm and snug in our pockets and handbags.

Hence, the present study was undertaken to determine the bacterial flora on mobile phones of HCWs \& their antimicrobial susceptibility pattern.

Out of 150 cell phones of HCWs, growth was observed in $141(94.05 \%)$ cell phones. Our study findings are similar to the findings of other studies done by Neha Sharma et al., ${ }^{8}$, Jayalakshmi et al., ${ }^{9}$, Ulger et al., ${ }^{10}$, and Sham et al., ${ }^{11}$ which showed positivity of $87 \%$, $91.6 \%, 94.5 \%$ and $99 \%$ respectively and it is in contrast to other studies done by Usha et al., ${ }^{12}$, Ramesh et al., ${ }^{13}$ and Kabir et al., ${ }^{14}$ which showed positivity of $40.62 \%, 45 \%$ and $62 \%$ respectively (Fig. 1 and 2 ). 
Table.1 Number of cell phones showing microbial growth before decontamination

\begin{tabular}{|c|c|c|c|}
\hline & Total samples & Positive growth & Percentage \\
\hline HCWs & 150 & 141 & $94.05 \%$ \\
\hline Non HCWs & 50 & 15 & $30 \%$ \\
\hline
\end{tabular}

Table.2 Comparison of microbial growth on cell phones of HCWs and Non HCWs before decontamination

\begin{tabular}{|c|c|c|c|c|}
\hline \multirow{2}{*}{$\begin{array}{c}\text { Organisms } \\
\text { isolated }\end{array}$} & \multicolumn{2}{|c|}{ HCWs (T=141) } & \multicolumn{2}{c|}{ Non HCWs (T=15) } \\
\hline CONS & 113 & $80.14 \%$ & Number & Percentage \\
\hline $\begin{array}{c}\text { Gram positive } \\
\text { bacilli }\end{array}$ & 96 & $68.1 \%$ & 7 & $73.34 \%$ \\
\hline MSSA & 28 & $19.8 \%$ & -- & $46.67 \%$ \\
\hline MRSA & 24 & $17 \%$ & -- & -- \\
\hline Micrococcus & 14 & $9.9 \%$ & -- & -- \\
\hline Pseudomonas & 14 & $9.9 \%$ & -- & -- \\
\hline Enterococcus & 10 & $7.1 \%$ & -- & -- \\
\hline E coli & 7 & $4.9 \%$ & -- & -- \\
\hline
\end{tabular}

Table.3 Comparison of microbial growth on cell phones of HCWs and Non HCWs after decontamination

\begin{tabular}{|c|c|c|c|c|}
\hline \multirow{2}{*}{ Organisms isolated } & \multicolumn{2}{|c|}{ HCWs (T=15) } & \multicolumn{2}{c|}{ Non HCWs (T=0) } \\
\cline { 2 - 5 } & Number & Percentage & Number & Percentage \\
\hline CONS & 11 & $73.34 \%$ & 0 & 0 \\
\hline Gram positive bacilli & 7 & $46.67 \%$ & 0 & 0 \\
\hline MSSA & 4 & $26.67 \%$ & 0 & 0 \\
\hline MRSA & 2 & $13.34 \%$ & 0 & 0 \\
\hline
\end{tabular}

Table.4 Antibiotic sensitivity pattern of Gram positive organisms isolated from mobile phones of HCWs

\begin{tabular}{|c|c|c|c|c|c|c|c|c|c|c|c|c|c|}
\hline Organisms & $\begin{array}{c}\text { No. of } \\
\text { isolates }\end{array}$ & CX & AK & GEN & AMC & CTX & CIP & E & CD & DO & LZ & VA & TEI \\
\hline CONS & 113 & $80 \%$ & $73 \%$ & $80 \%$ & $85 \%$ & $80 \%$ & $85 \%$ & $73 \%$ & $75 \%$ & $79 \%$ & $100 \%$ & $100 \%$ & $100 \%$ \\
\hline $\begin{array}{c}\text { Gram positive } \\
\text { bacilli }\end{array}$ & 96 & $76 \%$ & $75 \%$ & $68 \%$ & $75 \%$ & $82 \%$ & $84 \%$ & $80 \%$ & $81 \%$ & $76 \%$ & $100 \%$ & $100 \%$ & $100 \%$ \\
\hline MSSA & 28 & $100 \%$ & $57 \%$ & $50 \%$ & $75 \%$ & $71 \%$ & $64 \%$ & $50 \%$ & $64 \%$ & $71 \%$ & $100 \%$ & $100 \%$ & $100 \%$ \\
\hline MRSA & 24 & $0 \%$ & $34 \%$ & $29 \%$ & $37 \%$ & $25 \%$ & $46 \%$ & $21 \%$ & $50 \%$ & $58 \%$ & $100 \%$ & $79 \%$ & $100 \%$ \\
\hline Micrococcus & 14 & $79 \%$ & $71 \%$ & $86 \%$ & $86 \%$ & $79 \%$ & $64 \%$ & $86 \%$ & $100 \%$ & $79 \%$ & $100 \%$ & $100 \%$ & $100 \%$ \\
\hline Enterococcus & 10 & $50 \%$ & $70 \%$ & $60 \%$ & $60 \%$ & $60 \%$ & $40 \%$ & $50 \%$ & $100 \%$ & $50 \%$ & $100 \%$ & $100 \%$ & $100 \%$ \\
\hline
\end{tabular}

CX - Cefoxitin, AK - Amikacin, GEN - Gentamicin, AMC - Amoxicillin/Clavulanate, CTX - Cefotaxime, CIP -

Ciprofloxacin, E - Erythromycin, CD - Clindamycin, DO - Doxycycline, LZ - Linezolid, VA - Vancomycin, TEI -

Teicoplanin 
Table.5 Antibiotic sensitivity pattern of Gram negative organisms isolated from mobile phones of HCWs

\begin{tabular}{|c|c|c|c|c|c|c|c|c|c|c|c|c|}
\hline Organisms & $\begin{array}{c}\text { No. of } \\
\text { isolates }\end{array}$ & AK & GEN & AMC & CTX & CAZ & CXM & CIP & IMP & PI & TOB & CL \\
\hline Pseudomonas & 14 & $36 \%$ & $36 \%$ & $64 \%$ & $71 \%$ & $64 \%$ & $71 \%$ & $36 \%$ & $100 \%$ & $100 \%$ & $100 \%$ & $100 \%$ \\
\hline E coli & 07 & $43 \%$ & $29 \%$ & $71 \%$ & $57 \%$ & $57 \%$ & $43 \%$ & $43 \%$ & $100 \%$ & $100 \%$ & $100 \%$ & $100 \%$ \\
\hline
\end{tabular}

CAZ - Ceftazidime, CXM - Cefuroxime, IMP - Imipenem, PI - Piperacillin, TOB - Tobramycin, CL - Colistin

Table.6 Antibiotic sensitivity pattern of microorganisms isolated from mobile phones of Non

HCWs

\begin{tabular}{|c|c|c|c|c|c|c|c|c|c|c|c|c|c|}
\hline Organisms & $\begin{array}{c}\text { No. of } \\
\text { isolates }\end{array}$ & CX & AK & GEN & AMC & CTX & CIP & E & CD & DO & LZ & VA & TEI \\
\hline CONS & 11 & $91 \%$ & $91 \%$ & $91 \%$ & $100 \%$ & $91 \%$ & $73 \%$ & $73 \%$ & $100 \%$ & $100 \%$ & $100 \%$ & $100 \%$ & $100 \%$ \\
\hline $\begin{array}{c}\text { Gram positive } \\
\text { bacilli }\end{array}$ & 07 & $86 \%$ & $86 \%$ & $86 \%$ & $100 \%$ & $86 \%$ & $71 \%$ & $71 \%$ & $86 \%$ & $86 \%$ & $100 \%$ & $100 \%$ & $100 \%$ \\
\hline
\end{tabular}

Table.7 Efficacy of decontamination with a single 70\% isopropyl alcohol swipe

\begin{tabular}{|c|c|c|}
\hline & $\begin{array}{c}\text { No. of cell phones of HCWs } \\
\text { showing microbial growth }\end{array}$ & $\begin{array}{c}\text { No. of cell phones of Non HCWs } \\
\text { showing microbial growth }\end{array}$ \\
\hline Before decontamination & 141 & 15 \\
\hline After decontamination & 15 & 0 \\
\hline Efficacy of 70\% isopropyl alcohol & $89 \%$ & $100 \%$ \\
\hline
\end{tabular}

Fig.1 Comparison of microbial growth of mobile phones of HCWs \& Non HCWs before decontamination

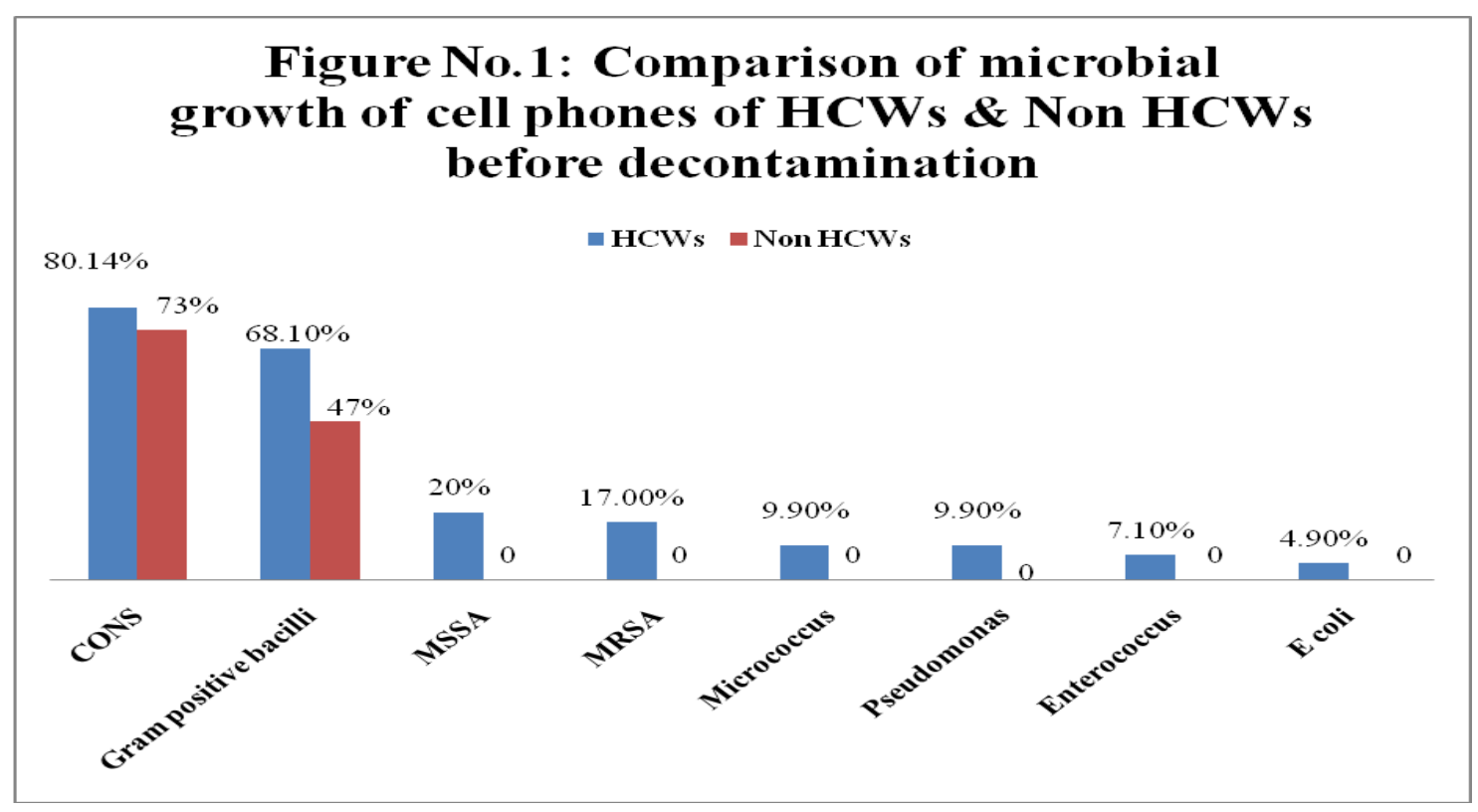


Fig.2 Microbial growth on cell phones of HCWs before decontamination

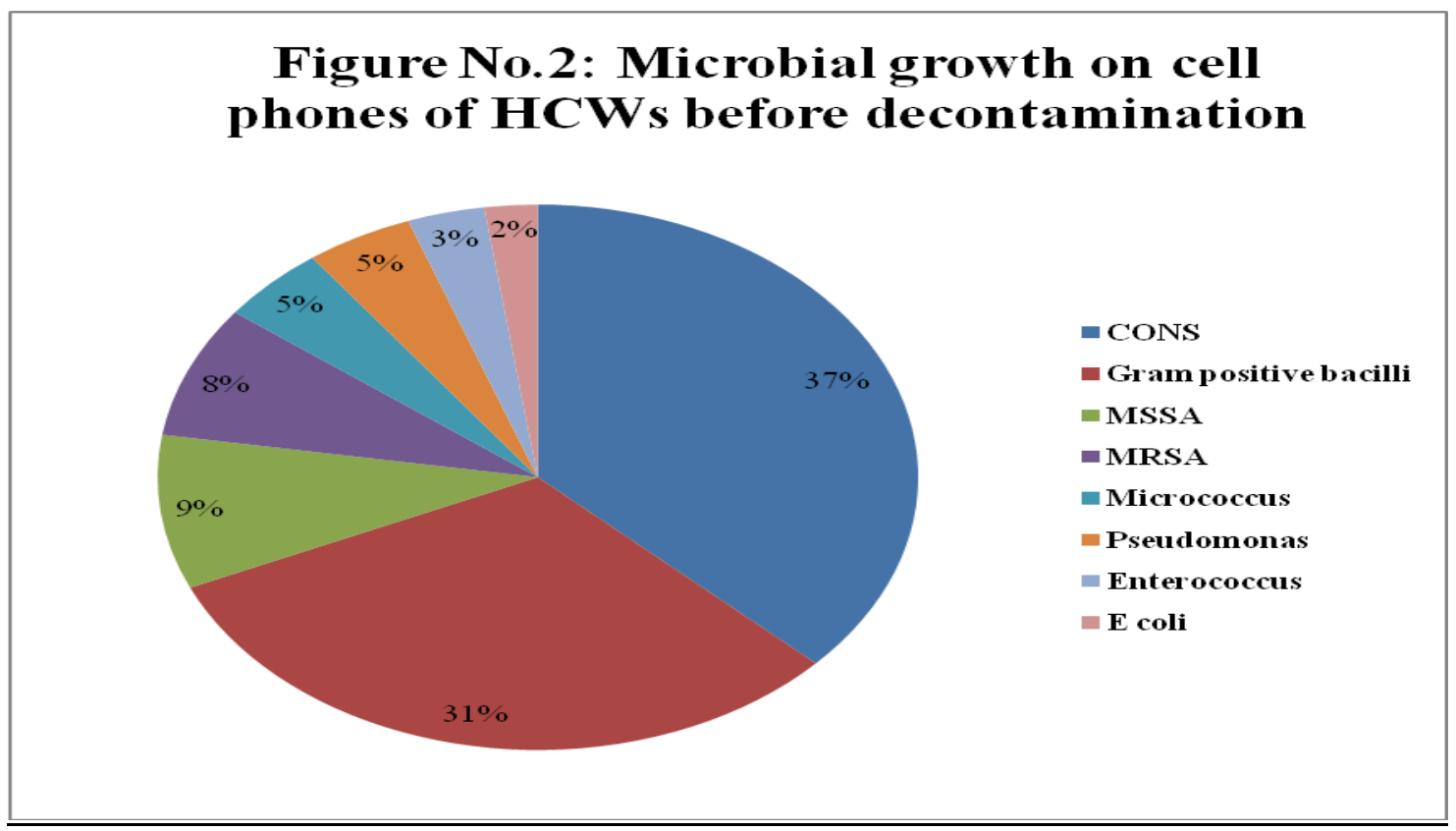

In HCWs, a total of 306 bacterial isolates were isolated of which Coagulase negative staphylococcus $(113 ; 80.14 \%)$ was the most predominant organism, followed by Gram positive bacilli (96; 68.1\%), Methicillin sensitive Staphylococcus aureus (28; 19.8\%), Methicillin resistant Staphylococcus aureus (24; 17\%), Micrococcus (14; 9.9\%), Pseudomonas (14; 9.9\%), Enterococcus (10; $7.1 \%)$ and $E$. coli $(7 ; 4.9 \%)$.

In our study CONS was the most common organism in HCWs which goes well with the studies of Usha et al., ${ }^{12}$, Jayalakshmi et al., ${ }^{9}$, Harish Trivedi et al., ${ }^{15}$ and Kabir et al., ${ }^{14}$. Although CONS is a normal skin flora responsible for a large number of hospital acquired infections but often proves difficult to treat because of the bacterium's genetic characteristics and growing resistance to highpowered antibiotics ${ }^{15}$.

In our study MRSA was isolated in cell phones of HCWs which correlates well with the results obtained by Jayalakshmi et al., ${ }^{9}$ and Usha et al., ${ }^{12}$ who have shown that MRSA was isolated only from the cell phones of HCWs. The presence of E. coli suggest fecal contamination of mobile phones which can result in community acquired infections and disease outbreaks.

However, out of 50 cell phones of non HCWs, growth was observed in only $15(30 \%)$ cell phones, of which $11(73.34 \%)$ were CONS, $7(46.67 \%)$ gram positive bacilli and the remaining were sterile. In other studies done by Usha et al., ${ }^{12}$ and Datta et al., ${ }^{16}$ in Non HCWs, growth was observed in $21.25 \%$ and $10 \%$ respectively.

Antibiotic susceptibility testing (AST) indicates marked resistance of bacterial isolated to commonly used antibiotics. This could be attributed to over the counter sale of antibiotics without any medical prescription leading to antibiotics abuse and high antibiotic resistance.

A simple procedure of decontaminations with a single $70 \%$ isopropyl alcohol swipe was done on both HCWs and non HCWs mobile 
phones. The efficacy of decontamination was found to be $89 \%$ as only 15 cell phones showed growth consisting of CONS(11;73.34\%), followed by Gram positive bacilli(7;46.67\%), MSSA(4;26.67\%), $\operatorname{MRSA}(2 ; 13.34 \%)$ among HCWs \& $100 \%$ among non HCWs. Similar results were shown by studies done by Neha Sharma et al., ${ }^{8}$ and Usha A et al., ${ }^{12}$ who got efficacy of $80 \%$ and $98 \%$ respectively by using $70 \%$ isopropyl alcohol for mobile decontamination.

Organisms like Staphylococcus aureus and coagulase negative staphylococcus resist drying and thus can survive and multiply rapidly in the warm environments like cell phones. Furthermore, formation of one biofilm by one bacterial agent can affect the survival of other pathogens on the same surface ${ }^{17}$.

The higher rates of contamination of cell phones in HCWs in our study might be due to the influence of various factors like general hygiene and hand washing practices of the HCWs, disinfection practices, frequency of use and cleaning of cell phones etc. Also, there are no guidelines for the care, cleaning and restriction of mobile phones in our health care settings. Thus the mobile phones may serve as mechanical vectors for transmission of multi drug resistant organisms to patients and even to their family members. Hand washing is the simplest and most economical measure that can prevent transfer of harmful pathogens. Periodic decontamination of the mobile phones with alcohol disinfectant may reduce the risk of cross contamination caused by mobile phones. Other measures include staff education, restricting use of mobile phones in high risk areas in hospital like intensive care units, operation theatres, burn units etc.

We conclude that mobile phones of HCWs can act as potential source to spread nosocomial infections as they were highly contaminated with various multidrug resistant bacteria. We also conclude that a single $70 \%$ isopropyl alcohol swipe can reduce the risk of contamination of mobile phones. Since restriction on the use of mobile phones by HCWs in hospital is not a practical solution, we recommend simple measures like hand washing after examining each patient, using hand free mobile phone during working hours, periodic cleaning of mobile phones with $70 \%$ isopropyl alcohol, a good hospital infection control programme and creating awareness in HCWs as well as non HCWs for proper handling of mobile phones to reduce rate of HAIs. Mobile phones are a boon for communication but it is up to us not to make it a bane.

\section{References}

Dardi Charan KG, Jaishree SP. Study of microorganisms and their antimicrobial susceptibility pattern on mobile phones of health care workers from a tertiary care rural hospital. Advances in Biomedicine and Pharmacy 2015; 2(6): 267-273.

Hassan AK, Fatima KB, Riffat M. Nosocomial infections: Epidemiology, prevention, control and surveillance. Asian Pacific Journal of Tropical Biomedicine 2017; 7(5): 478-482.

Collee J G, Miles R S, Watt B. Tests for identification of bacteria In: Colle $\mathrm{J} \mathrm{G}$, Duguid JP, Frase AG, Marmion BP Mackie, McCartney (Edt). Practical medical microbiology.14th ed., Vol 2 Churchill Livingstone, London 1996.pp.131-48.

Performance Standards For Antimicrobial Disc Susceptibility Tests. CLSI 2007; 25(1): 1.

WHO/UNICEF Joint monitoring Programme for water supply and sanitation. 2013. "Progress on sanitation and drinking water - 2013 update", Technical report.

Cell phones dirtier than a toilet seat. Accessed on, Aug. 2010, Available from 
http://news.softpedia.com/news/Cellpho nes-Dirtier-Than-a-Toilet-seat44854.shtml

Neha S, Arun S, Parihar RS, Khatri PS, Arvind C, Archana B. Prevalence and antibiotic pattern of microbes isolated from mobile phones of health care workers and non health care workers. International Journal of Current Microbiology and Applied Sciences 2014; 3(2): 43-60.

Jayalakshmi J, Appalaraju B, Usha S. Cellphones as reservoirs of nosocomial pathogens. Journal of the Association of Physicians of India 2008; 56: 388-389.

Ulger F, Esen S, Dilek A, Yanik K, Gunaydin M, Leblebicioglu H. Are we aware how contaminated our mobile phones with nosocomial pathogens? Ann Clin Microbiol Antimicrob 2009;8:7.

Sham SB, Sundeep HK, Shailaja KS. Potential of mobile phones to serve as a reservoir in spread of Nosocomial pathogens. Online Journal of Health and allied sciences 2011; 10(2): 14-16.

Usha A, Pushpa D, Aarti C, Sita M. Cellphones a modern stayhouse for bacterial pathogens. JK Science 2009; 11(3): 127 129.

Ramesh J, Carter AO, Campbell MH, Gibbons N, Powlett C, Moseley H.Sr., Levis D,
Carter T. Use of mobile phones by medical staff at Queen Elizabeth Hospital Barbados: evidence for both benefit and harm. J Hospt Infect 2008;70:160-165.

Kabir OA, Audu DA, Olabisi OA. The potential role of mobile phones in the spread of bacterial infections. J Infect Dev Ctries 2009;3(8):628-632.

Harish RT, Kairavi JD, Lopa PT, Saklainhaider SM, Tanuja BJ. Role of mobile phone in spreading hospital acquired infection: A study in different group of health care workers. National Journal of Integrated Research in Medicine 2011; 2(3): 61-66.

Datta P, Bansal N, Chander J. Hand held hazards by health care workers. Indian Journal of Medical Microbiology 2015; 31(3): 320-321.

Hassan AN, Birt DM, Frank JF. Behavior of Listeria monocytogenes in a pseudomonas putida biofilm on a condensate forming surface. J Food Prot 2004; 67(2): 322-327.

Wikipedia, The free Encyclopedia. Updated 02 October 2019,16:13 UTC. Available from https://en.wikipedia.org/wiki/List of countries by number of mobile phones in use. Retrieved 07 November 2019.

\section{How to cite this article:}

Siddesh Basawaraj Sirwar and Javeria Firdous. 2019. Mobile Phones of Healthcare Workers A Boon for Communication or A Potential Reservoir of Nosocomial Pathogens? Int.J.Curr.Microbiol.App.Sci. 8(11): 1779-1786. doi: https://doi.org/10.20546/ijcmas.2019.811.209 